\title{
Les Difficultés D'apprentissage En Éducation Physique Et Sportive: Une Étude De Trois Cas D'enseignement De La Course De Haies Au Bénin
}

\author{
Agbodjogbe D. Basile \\ Ogueboule Bachar M. \\ Attiklemé Kossivi
}

Laboratoire de Didactique des Disciplines (LDD), Institut National de la Jeunesse, de l'Education Physique et du Sport (INJEPS),

Université d'Abomey-Calavi (UAC), Bénin

\section{Kpazaï Georges}

Laboratoire de recherche sur l'intervention en activité physique (LARIAP),

École des Sciences de l'Activité Physique,

Université Laurentienne, Sudbury (ON), Canada

Doi:10.19044/esj.2018.v14n31p370 URL:http://dx.doi.org/10.19044/esj.2018.v14n31p370

\begin{abstract}
This study explored the functioning of the in-situ didactic system to highlight the difficulties experienced by students in the third year of the secondary school level when performing the activities suggested by their Sport and Physical Education Teachers. Based on the logic of the ternarity of the didactic system (Amade-Escot, 2008; 2003), the interactions between the teachers and students during the teaching/learning of the race were the subjects of study. A triangulation method was utilized whereby data was collected by extracting information from various sources including (Leutenegger, 2009; Van Der Maren, 1996): documentary analysis, interviews conducted pre-session, post-session, and post-cycle, as well as audio-visual recording of the class sessions. Central concepts within the anthropological theory of didactic (Chevallard, 1992) and didactic joint action theory (Sensevy, 2007) were used to analyze and interpret the results of which the most salient revealed that the students have difficulty with crossing and resuming the race after the hurdle. The inter-hurdle race does not occur at regular intervals. These are difficulties previously noted by Lamote (2002) and Aubert (1994). Within the context of our investigation, the agglomeration of the contents of the program, the number of sessions (03) devoted to the teaching/learning cycle of this physical activity, the quality of the material used, and the personal interactions between the
\end{abstract}


participants, were all factors shown to account for the difficulties experienced by the students.

Keywords: Hurdle race - Difficulties - Learning-Didactic interaction Triangulation.

\section{Résumé:}

La présente étude a exploré le fonctionnement du système didactique in-situ pour attirer l'attention sur les difficultés des élèves de la 3e année du niveau secondaire qui exécutent les tâches et activités proposées par des enseignants d'éducation physique et sportive (EPS) lors du processus enseignement-apprentissage. Dans la logique de la ternarité du système didactique (Amade-Escot, 2008 ; 2003), les enseignants et leurs élèves en interaction ont été les sujets d'étude. Pour répondre aux besoins de triangulation (Leutenegger, 2009; Van Der Maren, 1996), plusieurs techniques de collecte de données furent utilisées: une analyse documentaire, des entretiens anté-séance, post-séance, post-cycle, et des enregistrements audio-visuels des séances d'enseignement. Des concepts mobilisés par la théorie anthropologique du didactique (Chevallard, 1992) et la théorie de l'action didactique conjointe (Sensevy, 2007) ont été utilisés pour analyser et interpréter les résultats dont les plus saillants révèlent que les élèves ont des difficultés au franchissement et à la reprise de la course après la haie. La course inter-haie n'est pas régulière. Ce sont des difficultés déjà relevées par Lamote (2002) et Aubert (1994). Dans notre milieu d'investigation, le méli-mélo au niveau des contenus du programme, le peu de nombre de séances (03) consacré au cycle d'enseignement/apprentissage de cette APS, la qualité du matériel utilisé et le rapport personnel des interactants permettent d'expliquer les difficultés des élèves.

Mots-clés : Course de haies - Difficultés - Apprentissage-Interaction didactique -Triangulation.

\section{Introduction :}

\section{Contexte institutionnel de la réforme au Bénin}

Depuis l'accession du Bénin à l'indépendance en 1960, son système éducatif a continuellement subi de multiples reformes conduisant à des modifications de programmes (Oguéboulé, 2008; Zomanhoun, 2008 ; Agbodjogbé, 2007). La mise en œuvre des programmes du secondaire en général et ceux de l'Education Physique et Sportive (EPS) en particulier est actuellement fondé sur une approche qui semble être adaptée à la nouvelle mission assignée à l'école béninoise. C'est une vision qui a réactualisé les actions pédagogiques en EPS avec une planification plus harmonieuse de 
l'enseignement de cette discipline dont la légitimité semble être mal en appoint (Ministère des Enseignements Primaire et Secondaire, 2007, p 3). Des documents guide et programme ont commencé par être édités par le Ministère en charge de l'enseignement secondaire général à partir de 2002 (Attiklémè, 2009). Selon Attiklémè et Kpazaï (2011), ils visent l'enseignement de toutes les disciplines inscrites dans l'emploi du temps de l'élève selon l'Approche Par les Compétences (APC). C'est une approche curriculaire fondée sur deux paradigmes épistémologiques principaux : le constructivisme et le socioconstructivisme. Elle est axée sur une nouvelle démarche, celle de la résolution de problème (Jonnaert, 2002). Ainsi, dans cette logique, le contenu du programme de formation au Bénin vise à former " un citoyen autonome, intellectuellement et physiquement équilibré, capable d'entreprendre, de se prendre en charge et d'apprendre tout au long de sa vie ; un citoyen respectueux de la personne, de la vérité et de la démocratie, animé d'un esprit de méthode, de coopération et du goût de la responsabilité ; un citoyen gestionnaire de lui-même, gestionnaire de l'environnement et gestionnaire des situations de la vie sociale » (Ministère de l'Education Nationale, 2007, p. 4-5). L'enseignement de l'EPS a pour but de contribuer à la formation de ce type de béninois. Par ailleurs, le troisième article de la charte internationale de l'UNESCO (1978) sur l'Éducation Physique et le Sport stipule que leur enseignement doit répondre aux besoins de l'individu et de la société.

En EPS, les Activités Physiques et Sportives (APS) déterminent les éléments de la culture que l'élève doit maîtriser à la suite d'une progression pédagogique (Jeannin, 2011). La refonte du système éducatif a conduit à l'élaboration des programmes impliquant une méthode d'enseignement centrée sur l'élève. La mise en application de cette méthode devrait rendre l'élève autonome et capable de s'intégrer dans la société. Selon Legendre (1993), l'enseignement est un processus de communication en vue de susciter l'apprentissage. Au sens didactique du terme, Marsenach et Mérand (1987) précisent qu'en EPS, l'enseignant n'enseigne que lorsqu'il régule l'activité des élèves aux prises avec une tâche d'apprentissage. Il ressort de ces définitions qu'enseigner en EPS consisterait à transformer la conduite motrice des élèves avec des méthodes appropriées par le truchement des APS. L'APS qui est l'objet de notre étude est la course de haies.

La course de haies est une épreuve dont la logique et le défi consistent à essayer de courir le plus vite possible malgré la présence d'obstacles. Ces obstacles - les haies - représentent des contraintes qui exigent l'adaptation d'une motricité particulière, la mobilisation de ressources de façon bien spécifique. La contrainte principale est représentée par le rapport entre la longueur des intervalles et la hauteur des haies (Aubert, 1994). Plus les haies sont hautes et rapprochées, plus l'épreuve est difficile. Des contraintes 
supplémentaires proviennent du nombre de haies à franchir et de la longueur du parcours.

Quatre sections structurent cet article. Après avoir présenté le contexte institutionnel de la réforme et situé l'article dans son contexte, nous présenterons l'inscription théorique de l'étude qui débouchera sur la problématique, la question et l'hypothèse de la recherche. La troisième section abordera la démarche méthodologique utilisée. La quatrième partie s'attachera à montrer à partir de l'analyse de quelques transactions didactiques les difficultés de l'implémentation de la course de haies au collège OKEDAMA de Parakou en république du Bénin.

\section{Vers une problématisation de l'étude}

Conformément aux programmes d'étude de l'EPS en vigueur au Bénin, la course de haies s'enseigne en classe de $4^{\text {e }}$ et de $2^{\text {nd }}$. Les travaux de Bankolé (2013) ont montré que l'enseignement de cette APS est problématique. A la fin des trois séances de classe réservées à son enseignement, plusieurs élèves présentent encore des difficultés aussi bien dans l'exécution correcte des foulées inter-haies que dans le franchissement des haies. Ce constat qui doit être établi scientifiquement suppose qu'il y a des faits qui entravent la réussite de l'apprentissage et l'acquisition des techniques de cette APS au niveau des élèves. C'est pour cette raison, que nous organisons nos réflexions autour de l'enseignement/apprentissage de la course de haies dans les lycées et collèges. L'objectif de l'enseignant est d'améliorer les habiletés motrices de l'élève à travers plusieurs types de ressources que ce dernier devrait solliciter. Ces ressources sont principalement d'ordre technique (pour un bon passage de haies) et biomécanique (pour une utilisation efficace du corps dans l'action motrice) selon Hubiche et Pradet (2000). Ces caractéristiques justifieraient la complexité de l'enseignement/apprentissage de la course de haies en milieu scolaire.

\subsection{La problématique, la question de recherche et l'hypothèse}

Depuis l'effectivité de l'APC en situations ordinaires de classe en EPS, de nombreux travaux ont focalisé leur attention sur les pratiques enseignantes. Les études menées par Agbodjogbé (2013 ; 2007), Oguéboulé (2008) et Atoun $(2016 ; 2005)$ ont montré et expliqué les transpositions didactiques opérées par les enseignants, confrontés à des difficultés dans la mise en œuvre de la démarche d'enseignement/apprentissage/évaluation. De façon plus explicite, des auteurs se sont intéressés aux adaptations des contenus prescrits, opérées par les enseignants dans leur milieu d'intervention, pour le cas de certaines APS (voir entre autres les travaux de: Oguéboulé, Agbodjogbé, Attiklémè, Kouété et Kpazaï (2016) sur 
l'enseignement du lancer de javelot; Atoun, Agbodjogbé, Attiklémè, Oguéboulé et Kpazaï (2015) sur l'évaluation de fin de cycle au volleyball; Bankolé (2013), à propos de la course de haies; Gangbadja (2013), à la course de relais; Agassounon (2013), en ce qui concerne le basketball; Boni (2011), sur la gymnastique; Zinsou (2011), au volleyball; Fagnon (2010), au saut en longueur; Agbotouédo (2008), sur la course de vitesse; Abaya (2004), pour le cas de football). Ce sont des travaux qui ont mis l'accent sur la démarche d'enseignement et les contenus de savoir proposés aux élèves sans porter un regard sur les difficultés rencontrées par ces derniers lors de l'apprentissage. Ces difficultés peuvent induire des écarts entre les contenus enseignés et ceux réellement appris. La situation ordinaire de classe impose une pratique relationnelle, voire dialogique entre l'enseignant et ses élèves ; elle est finalisée par l'apprentissage de savoirs et savoir-faire socialement et culturellement identifiés (Martinand, 1989; Perrenoud, 1998; Poussin, 2009). Ce qui signifie qu'en classe, les élèves et l'enseignant forment avec le savoir en jeu, le système didactique (Amade-Escot, 2008, 2003; Amade-Escot, Garnier et Monnier, 2007; Chevallard (1992 ; 1991) et Sensevy (2007 ; 2006). Mais avant la situation de classe, les savoirs savants, les pratiques en cours dans la société (pratiques sociales de référence) comme les APS (la course de haies par exemple) subissent des transformations pour devenir des objets à enseigner par le biais des programmes d'étude (Chevallard, 1992; Martinand, 1989 ; Odjoussou, 2010 ; Perrenoud, 1998 ; Verret, 1975). Ces transformations qui continuent en situation de classe peuvent être liées aux difficultés rencontrées par les élèves.

En admettant que les élèves et l'enseignant n'occupent pas la même position (topogenèse) dans l'institution classe (Chevallard, 1992; 1991; Sensevy, 2007 ; 2006), il nous est paru important de relever et d'analyser les difficultés des élèves exécutant les tâches et activités d'apprentissage proposées par les enseignants. En accord avec les résultats de Bankolé (2013) qui stipulent que les difficultés d'enseignement de la course de haies sont relatives aux rapports personnel et institutionnel de l'enseignant à l'APS et à la démarche prescrite, nous avons choisi la même APS pour explorer l'instance du système ternaire que sont les élèves sans toutefois extirper cette instance dans ses rapports avec les autres.

C'est dans la logique de cette problématique que s'inscrivent notre question de recherche et la proposition de réponse que nous avons émise.

\section{Question de recherche}

La question essentielle de nos investigations s'articule autour des difficultés rencontrées par les élèves dans la réalisation des gestes techniques de la course de haies et leurs causes. Elle est intitulée de la façon suivante : 
Comment peut-on expliquer les difficultés rencontrées par les élèves lors de l'apprentissage de la course de haies ?

\section{Hypothèse}

Les difficultés rencontrées par les élèves lors de l'apprentissage de la course de haies sont relatives à la course inter-haies et au franchissement. Le rapport personnel des acteurs du système didactique (élèves et enseignants) à la course de haies, le nombre de séances consacré à cette APS, l'inadéquation du matériel aux activités d'apprentissage proposées par les enseignants permettent d'expliquer les difficultés des élèves. Pour vérifier nos assertions, une démarche méthodologique a été mise en place.

\section{La méthodologie}

\subsection{Le cadre de l'étude}

Pendant la période de nos investigations (année scolaire 2014-2015), le Ministère de l'Enseignement Secondaire, de la Formation Technique et Professionnelle, de la Reconversion et de l'Insertion des Jeunes (MESFTPRIJ) est chargé de gérer les collèges d'enseignement général (CEG) et les lycées de formations techniques et professionnelles. Avec des attributions plus réduites, cet d'ordre d'enseignement est devenu depuis avril 2017, le Ministère de l'Enseignement Secondaire, Technique et de la Formation Professionnelle (MESFTP).

Il y est remarqué une grande pénurie d'enseignants et surtout en EPS. Cela fait que certaines classes sont dispensées des cours d'EPS. Pour l'année scolaire 2013-2014, sur un total de 8853 enseignants (Agents permanents et contractuels de l'Etat) en situation de classe, seulement 274 sont des enseignants d'EPS, soit un pourcentage de 3,09\% (Direction de l'Enseignement Secondaire Général, 2014). Au titre de la même année scolaire, sur un total 768175 élèves inscrits dans le secondaire général public, seulement15847groupes pédagogiques ont été constitués. En 20142015, l'effectif des élèves du public est passé à 805017 au Bénin. De plus, les problèmes de manque d'infrastructures sportives et de matériels pédagogiques influent sur la qualité de l'enseignement/apprentissage. C'est dans ce contexte que se déroulent les cours d'EPS.

\subsubsection{Le format d'enseignement prescrit par les Nouveaux Programmes d'Enseignement (NPE)-EPS au Bénin}

Le programme d'étude selon l'APC en EPS s'articule autour de deux Situations d'Apprentissage (SA). La première SA, intitulée «Pratiquer des activités physiques de maîtrise de corps et de l'environnement», est consacrée à l'enseignement des sports individuels. Quant à la deuxième SA, intitulée «Pratiquer des activités physiques de coopération et/ou 
d'opposition ", elle est destinée à l'enseignement des jeux sportifs collectifs et des luttes. Chaque SA se déroule en douze séances de classe de trois heures hebdomadaires chacune (trois séances au moins par APS). Neuf séances sont réservées pour l'enseignement/apprentissage de trois à quatre APS ; il est prévu une séance bilan et deux séances pour l'évaluation sommative des apprentissages marquant la fin du cycle. Dans la logique du projet d'EPS d'établissement, les enseignants adaptent parfois le programme aux réalités du terrain. En classe de $4^{\mathrm{e}}$, la SA $\mathrm{N}^{\circ} 1$ prévoit l'enseignement de la course de haies, du saut en hauteur, de la gymnastique au sol et du grimper à la corde. Les sports collectifs tels que, le handball, le volleyball et la lutte traditionnelle sont prévus pour être enseignés en $\mathrm{SA} \mathrm{N}^{\circ} 2$ (Ministère de l'Enseignement Secondaire, de la Formation Technique et Professionnelle, de la Reconversion et de l'Insertion des Jeunes, 2015). Dans cette classe, la distance à parcourir à la course de haies est de $100 \mathrm{~m}$ avec une largeur des couloirs qui est de $1,22 \mathrm{~m}$. Pour enseigner cette APS, la hauteur des haies est prévue pour être à $0,91 \mathrm{~m}$ chez les garçons et à $0,76 \mathrm{~m}$ chez les filles. La distance entre le départ et la première haie est de $13 \mathrm{~m}$ et celle inter-haies est de $8 \mathrm{~m}$ pour les filles. Pour les garçons, $14 \mathrm{~m}$ séparent le départ de la première haie et l'arrivée est à $8,5 \mathrm{~m}$ de la dernière haie.

\subsubsection{Description du contexte de l'étude}

Les données de terrain ont été collectées au CEG Okédama à Parakou. La ville de Parakou est située au centre de la République du Bénin à $407 \mathrm{~km}$ de Cotonou. Elle compte 10 établissements publics d'enseignement secondaire général dont le CEG Okédama, situé dans le $3^{\mathrm{e}}$ arrondissement, juste à l'entrée de la ville.

Le collège dispose de 21 groupes pédagogiques pour 4 enseignants d'EPS. Il y a 6 classes de $4^{\mathrm{e}}$ ordonnées A, B, C, D, E et F. Notons que les élèves de la classe de $6^{\mathrm{e}}$ ne font pas les cours d'EPS, toujours pour les mêmes raisons évoquées ci-dessus. Avec un effectif de 69 élèves, la $4^{\mathrm{e}} \mathrm{A}$ fait cours les lundis soirs de 16 heures à 19 heures. Les 68 élèves de la $4^{\mathrm{e}} \mathrm{C}$ font les cours d'EPS les mercredis matins de 7 heures à 10 heures. Dans ce même environnement didactique, la classe de $4^{\mathrm{e}} \mathrm{E}$ avec un effectif de 67 élèves, a son cours d'EPS les jeudis matins de 7 heures à 10 heures. Ces trois classes de $4^{\mathrm{e}}$ ont été prises en compte par notre étude.

Pour l'enseignement/apprentissage de la course de haies, le CEG Okédama possède des plots et une ficelle pour le tracer des couloirs de course; mais ne dispose d'aucune haie réglementaire. Les enseignants s'adaptent à cette difficulté en utilisant les pneus à la place des haies pour réaliser leurs cours. Les haies (pneus reliés par des bois placés horizontalement) sont rigides et ne sont pas modulables en termes de hauteurs. D'ailleurs les hauteurs sont inconnues et varient selon les pneus 
utilisés. Par contre, les haies réglementaires sont à des hauteurs connues et modulables selon les circonstances. C'est dans ce contexte que notre étude s'est intéressée à la problématique des difficultés liées à l'apprentissage de la course de haies dans les classes de $4^{\mathrm{e}}$ au CEG de Okédama.

\subsection{La nature et les sujets de l'étude}

Essentiellement de type qualitatif, la présente étude est basée sur l'analyse des phénomènes d'enseignement et d'apprentissage de la course de haies en situation ordinaire de classe. Dans cette logique, les sujets d'étude ne peuvent être que des enseignants d'EPS et leurs élèves pendant l'enseignement/apprentissage de la course de haies.

L'étude ayant eu lieu dans les classes de $4^{\mathrm{e}}$, nous disposons alors de 3 enseignants car le CEG ne contient que 3 classes de $4^{\mathrm{e}}$. Parmi ces enseignants participants à létude, il y a deux professeurs certifiés et un stagiaire en $5^{\mathrm{e}}$ année de formation pour devenir enseignant professeur certifié d'EPS.

Avec une expérience professionnelle d'enseignement de 7 ans, l'enseignant $\mathrm{E}_{1}$ a la $4^{\mathrm{e}} \mathrm{A}$ à sa charge tandis que l'enseignant $\mathrm{E}_{2}$, possédant 4 ans d'expérience professionnelle est chargé des cours d'EPS en $4^{\mathrm{e}} \mathrm{C}$. Le stagiaire, $\mathrm{E}_{3}$ est le professeur de la $4^{\mathrm{e}} \mathrm{E}$.

Dans la logique de la ternarité du système didactique (Amade-Escot, 2008 ; 2003; Sensevy, 2007 ; 2006), les élèves de $E_{1}$, $E_{2}$ et $E_{3}$ sont d'office sujets d'étude. Ne pouvant pas suivre tous les élèves à la fois, nous en avons choisi 4 par classe en référence aux travaux de Leutenegger (2009 ; 2003). Il s'agit pour chaque classe, des deux supposés être les plus forts et des deux supposés être les plus faibles. Ils sont choisis par l'enseignant qui est supposé connaître ses élèves. En définitive, 12 élèves et 3 enseignants d'EPS ont été investis en situation ordinaire avec des techniques et outils convenables.

\subsection{Les techniques et outils d'investigation}

Trois techniques ont été essentiellement utilisées et ont nécessité pour chacune d'elles, un certain nombre d'outils. Il s'agit de l'analyse documentaire, des entretiens et des enregistrements audio-visuels des séances.

\subsubsection{L'analyse documentaire}

Elle a été consacrée à la lecture des documents, des travaux qui existent et qui ont rapport avec la présente thématique. Le recours aux documents guides et programmes d'étude a permis de connaître et d'analyser les contenus définis en termes de savoirs et savoir-faire à enseigner dans les classes de $4^{\mathrm{e}}$ par le truchement des APS retenues, particulièrement la course 
de haies. Elle a abouti à la mise en tension des travaux menés sur cette APS, afin de faire émerger la pertinence de notre étude.

\subsubsection{Les entretiens}

A l'aide de guide d'entretien et de dictaphone, nous avons réalisé 3 types d'entretiens. Il s'agit des entretiens anté-séance et post-séance avec les enseignants puis des entretiens post-cycle avec les 12 élèves ciblés.

\subsubsection{Les entretiens anté-séance et post-séance}

Au nombre de 3 par enseignant, les 9 entretiens anté-séance ont eu lieu avant chaque séance. Ils ont mis chacun des trois enseignants en position de revenir sur la séance précédente et d'évoquer les objectifs poursuivis pour celle du jour. Les éventuelles difficultés que peuvent rencontrer les élèves lors de l'apprentissage des contenus de savoirs à leur enseigner et les solutions prévues pour y remédier ont été évoquées en référence aux travaux de Amade-Escot (2007; 2003) et de Vermersch (1994).

Conformément aux entretiens anté-séance, 3 entretiens post-séance ont été réalisés avec chaque enseignant. Ce qui fait un total de 9 entretiens postséance pour l'ensemble des 3 enseignants. Ils ont permis de faire, à chaud le bilan de chaque séance avec l'enseignant au sens de Leutenegger (2009; $2003)$ et Amade-Escot $\left(2008\right.$; 2003). Les enseignants $\mathrm{E}_{1}, \mathrm{E}_{2}$ et $\mathrm{E}_{3}$ sont donc revenus sur les savoirs et savoir-faire qu'ils ont enseignés, les tâches et activités d'apprentissage ayant créé des difficultés aux élèves et les solutions qu'ils ont proposées. Chacun des 18 entretiens (9 anté et 9 post) avec les professeurs a duré 10 minutes.

\subsubsection{Les entretiens post-cycle}

Ce sont des entretiens qui ont eu lieu avec des élèves à la fin de la troisième et dernière séance du cycle de l'enseignement/apprentissage de la course de haies. Ils sont au nombre de 12 conformément au nombre des élèves retenus et ont permis de recueillir des propos qui ont été confrontés à ceux des enseignants et aussi aux actions didactiques visualisées dans la logique de la triangulation évoquée par Van Der Maren (1996). Ainsi , les contenus de savoirs proposés par l'enseignant, les difficultés rencontrées par les élèves lors de l'exécution des tâches proposées et leur vécu à la course de haies en dehors des heures de cours d'EPS ont été abordés.

Les différents entretiens se sont déroulés dans un contexte de séances ordinaires de classe dont chacune a fait l'objet d'enregistrement audio-visuel. L'entretien avec chacun des 12 élèves a duré 5 minutes. 


\subsubsection{Les enregistrements audio-visuels des séances}

Pour faciliter les enregistrements audio-visuels, nous avons utilisé deux caméscopes munis chacun d'une caméra et d'un micro. Ce sont donc des observations instrumentées qui se sont focalisées surtout sur la phase de réalisation consacrée à la construction de nouveaux savoirs et savoir-faire par le professeur interagissant avec ses élèves. Au nombre de 3 par classe, un total de 9 séances ont donc été filmées et ont permis en référence aux travaux de Amade-Escot (2003), d'enregistrer les propos et les gestes des acteurs du système didactique dans le cycle consacré à l'enseignement/apprentissage de la course de haies en classe de $4^{\mathrm{e}}$ au CEG Okédama de Parakou au Bénin.

Un caméscope a été utilisé pour filmer l'ensemble de la classe et le second, beaucoup plus mobile a permis de filmer les centres particuliers d'intérêt qui sont notamment relatifs aux élèves ciblés. Les différents entretiens et observations ont eu lieu conformément à un chronogramme retenu de commun accord avec les enseignants $E_{1}, E_{2}$ et $E_{3}$.

\subsection{La retranscription et le traitement des données}

Conformément à notre démarche, les données ont été recueillies par analyse documentaire, entretiens et observations instrumentées. Si les données collectées au moyen de la lecture ont permis de faire la revue de littérature et de poser notre question de recherche, les entretiens ont été retranscrits verbatim. Ils sont par la suite regroupés en fonction des thématiques pour répondre à notre question de recherche.

Les informations issues de l'analyse des propos des différents acteurs du système didactique (enseignant et élèves) ont été confrontées pour plus d'objectivité.

Après plusieurs visualisations, chaque film de séance de classe à la course de haies a été retranscrit. Ces retranscriptions, en référence à DevosPrieur (2006) ont pris en compte le découpage du protocole selon les tâches proposées aux élèves par l'enseignant, les modalités de travail instaurées, les objets enseignés, les consignes du professeur et les conduites types des élèves. Les bandes vidéos ont facilité l'analyse du comportement des élèves aux prises avec les tâches proposées par les enseignants et qui induisent les difficultés rencontrées.

Pour une bonne interprétation des données collectées, des extraits de propos issus des entretiens avec les élèves et les enseignants ont servi de référence pour donner du sens aux informations recueillies par le biais des enregistrements audio-visuels des séances en accord avec Leutenegger (2009 ; 2003) et Van Der Maren (1996). Les retranscriptions de séances ont abouti à des corpus condensés des séquences de classe pour faciliter cette analyse et interprétation. 
C'est, cette démarche méthodologique qui a permis d'avoir les résultats que nous avons analysés et interprétés avec des concepts mobilisés par la théorie de l'anthropologie du didactique de Chevallard $(1992 ; 1991)$ puis celle de l'action conjointe didactique de Sensevy $(2007$; 2006) et Mercier, Schubauer-Leoni, Sensevy (2002).

\section{Présentation, analyse et discussion des résultats}

L'analyse des résultats issus de nos investigations est structurée en trois étapes ci-après:

a) les contenus de savoirs prévus pour être enseignés par les documents guide et programme de la classe de $4^{\mathrm{e}}$ à la course de haies ;

b) les contenus de savoirs proposés par les enseignants à leurs élèves en situation de classe à la course de haies ;

c) les difficultés rencontrées par les élèves lors de l'apprentissage de la course de haies et leurs causes éventuelles.

Pour parvenir à cette fin, nous avons utilisé essentiellement des tableaux suivis d'un commentaire.

\subsection{Les attendus du programme en terme de savoirs à la course de haies en classe de quatrième}

En référence au guide et au programme d'enseignement, nous avons récapitulé dans un tableau, les objets d'apprentissage de la course de haies en classe de $4^{\mathrm{e}}$. 


\begin{tabular}{|c|c|c|c|c|c|c|}
\hline & \multicolumn{5}{|c|}{$\begin{array}{l}\text { Tableau } 2 \text { : Récapitulatif des contenus à enseigner prévus par les documents guide et programme } \\
\text { Objets d'apprentissage }\end{array}$} & \multirow[b]{2}{*}{$\begin{array}{l}\text { Contenus } \\
\text { disciplinaires }\end{array}$} \\
\hline & Connaissances & Techniques & $\begin{array}{l}\text { Activités } \\
\text { d'apprentissage (AA) }\end{array}$ & $\begin{array}{l}\text { Procédure } \\
\text { d'apprentissage }\end{array}$ & $\begin{array}{l}\text { Stratégies } \\
\text { d'apprentissage }\end{array}$ & \\
\hline Guide & $\begin{array}{l}\text { Course de haies: } \\
\text { starter, départ } \\
\text { quadrupédique, } \\
\text { commandement, } \\
\text { signal de départ, } \\
\text { foulées entre les } \\
\text { haies, amplitude, } \\
\text { fréquence, attitude de } \\
\text { course, arrivée, } \\
\text { performance, hauteur } \\
\text { de la haie, intervalle } \\
\text { entre les haies. }\end{array}$ & $\begin{array}{l}\text { course de haie 100m: prise } \\
\text { de départ quadrupédique, } \\
\text { réaction aux } \\
\text { commandements et au } \\
\text { signal de départ, phase de } \\
\text { mise en action, arrivée, } \\
\text { mécanique de course, } \\
\text { attaque de la haie par la } \\
\text { montée du genou, passage } \\
\text { latéral de jambe d'appui, } \\
\text { reprise dynamique d'appui } \\
\text { derrière la haie, action } \\
\text { équilibratrice des bras, } \\
\text { régularité de la course, } \\
\text { mode de respiration, } \\
\text { franchir la ligne d'arrivée } \\
\text { en sprint }\end{array}$ & $\begin{array}{l}\text { AA n }{ }^{\circ} 1: \text { Franchir la } \\
\text { haie de manière efficace } \\
\text { - en marchant, } \\
\text { - en petites foulées, } \\
\text { - en courant ; } \\
\text { AAn } 2: \text { Réaliser une } \\
\text { course régulière et } \\
\text { accélérée entre les } \\
\text { haies ; } \\
\text { AAn } 3: \text { Attaquer la } \\
\text { première haie à une } \\
\text { grande vitesse }\end{array}$ & $\begin{array}{l}\text { Expression } \\
\text { Analyse } \\
\text { Planification } \\
\text { Exploration } \\
\text { Prise de position } \\
\text { Exécution } \\
\text { Objectivation } \\
\text { Auto-évaluation } \\
\text { Amélioration }\end{array}$ & $\begin{array}{l}\text { Brainstorming, } \\
\text { Travail individuel } \\
\text { Travail collectif } \\
\text { Travail en groupe } \\
\text { Enseignement par les } \\
\text { pairs } \\
\text { Exercice répétitif }\end{array}$ & \\
\hline Programme & $\begin{array}{l}\text { Idem aux contenus du } \\
\text { guide }\end{array}$ & Idem aux contenus du guide & $\begin{array}{l}\text { Parcourir les } 100 \mathrm{~m} \\
\text { haies de façon rapide et } \\
\text { coordonnée. }\end{array}$ & $\begin{array}{l}\text { Idem aux contenus du } \\
\text { guide }\end{array}$ & $\begin{array}{l}\text { Idem aux contenus du } \\
\text { guide }\end{array}$ & $\begin{array}{l}\text { 60m filles } \\
80 \mathrm{~m} \text { garçons }\end{array}$ \\
\hline
\end{tabular}


De l'analyse du tableau 2, il ressort que 1'enseignement de la course de haies en $4^{\mathrm{e}}$ est prévu pour être structuré autour de trois AA relatives chronologiquement au franchissement de la haie, à la course inter-haies et à l'attaque de la première haie. En analysant les prescriptions, on constate une ambiguïté à propos de la distance à parcourir. Les documents évoquent $60 \mathrm{~m}$ pour les filles, $80 \mathrm{~m}$ pour les garçons et aussi 100m sans distinction de sexe. Ce flou au niveau prescriptif peut créer d'embarras dans le choix des contenus de savoirs à enseigner par les professeurs (la distance à faire parcourir aux élèves par exemple). De plus, les connaissances et techniques sont listées pêle-mêle sans la moindre progression pédagogique. Tous les savoirs et savoir-faire relatifs à la course de haies sont prévus pour des élèves de $4^{\mathrm{e}}$ qui découvrent cette APS dans un contexte où, seulement trois séances sont consacrées à son enseignement.

\subsection{Les savoirs enseignés}

Les contenus des films ont été mis en tension avec l'analyse des différents entretiens pour inférer les savoirs proposés aux élèves par chaque enseignant dans son milieu d'intervention. Pour chaque séance de classe, un synopsis condensé a permis de récapituler la chronologie des savoirs et savoir-faire mis en jeu.

\subsubsection{Les savoirs mis à l'étude par l'enseignant $E_{1}$ : une difficile adaptation}

Chacune des trois séances de cet intervenant a été analysée pour faire émerger les savoirs réellement proposés aux élèves. Les tableaux 3, 4 et 5 ont servi de base d'analyse.

Tableau 3 : Synopsis condensé de la première séance déroulée par $E_{1}$.

\begin{tabular}{|c|c|c|c|}
\hline Contenus & Tâches & Capacités & Objectifs visés pour la séance \\
\hline \multirow{6}{*}{$\begin{array}{l}\text { Représentation } \\
\text { initiale de la } \\
\text { course de haies et } \\
\text { repérage du pied } \\
\text { d'appel }\end{array}$} & \multirow{3}{*}{$\begin{array}{l}\mathrm{N}^{\circ} 1: \text { Les } \\
\text { élèves disent et } \\
\text { font ce qu'ils } \\
\text { savent de la } \\
\text { course de haies. }\end{array}$} & Expression & $\begin{array}{l}\text { Evaluer les acquis antérieurs des élèves à } \\
\text { la course de haies }\end{array}$ \\
\hline & & Analyse & $\begin{array}{l}\text { Faire un récapitulatif des différentes } \\
\text { étapes de la réalisation de la course de } \\
\text { haies avec les élèves }\end{array}$ \\
\hline & & Planification & $\begin{array}{l}\text { Etablir un ordre d'apprentissage de ces } \\
\text { étapes : Repérage du pied d'appel- } \\
\text { Franchissement-Course inter haies-Reprise } \\
\text { de la course après le Franchissement. }\end{array}$ \\
\hline & \multirow{3}{*}{$\begin{array}{l}\mathrm{N}^{\circ} 2 \text { Repérage } \\
\text { du pied d'appel } \\
\text { en marchant, en } \\
\text { petites foulées } \\
\text { et en courant. }\end{array}$} & Exploration & $\begin{array}{l}\text { Chercher les voies et moyens pour repérer } \\
\text { son pied d'appel. }\end{array}$ \\
\hline & & $\begin{array}{l}\text { Prise de } \\
\text { position }\end{array}$ & $\begin{array}{l}\text { Identifier et dégager le bon geste pour en } \\
\text { faire un modèle d'exécution }\end{array}$ \\
\hline & & Exécution & $\begin{array}{l}\text { Attaquer la première haie avec la jambe } \\
\text { libre identifiée après une course accélérée. }\end{array}$ \\
\hline
\end{tabular}


Tableau 4 : Synopsis condensé de la deuxième séance déroulée par $\mathrm{E}_{1}$

\begin{tabular}{|c|c|c|c|}
\hline Contenus & Tâches & Capacités & Objectifs visés pour la séance \\
\hline \multirow{6}{*}{$\begin{array}{l}\text { Franchissement de } \\
\text { la haie et Course } \\
\text { rythmique dans } \\
\text { l'espace inter-haies. }\end{array}$} & \multirow{2}{*}{$\begin{array}{l}\mathrm{N}^{\circ} 1 \text { :Organises-toi avec } \\
\text { tes camarades pour } \\
\text { franchir les haies, jambe } \\
\text { d'attaque tendue et } \\
\text { perpendiculaire aux } \\
\text { lattes }\end{array}$} & $\begin{array}{l}\text { Exploration et } \\
\text { prise de position }\end{array}$ & $\begin{array}{l}\text { Identifier et dégager le bon } \\
\text { geste pour en faire un modèle }\end{array}$ \\
\hline & & Exécution & $\begin{array}{l}\text { Attaquer la haie, jambe } \\
\text { d'attaque tendue vers l'avant. }\end{array}$ \\
\hline & \multirow{2}{*}{$\begin{array}{l}\mathrm{N}^{\circ} 2: \text { Organises-toi } \\
\text { pour franchir les haies, } \\
\text { jambe d'esquive fléchie } \\
\text { et parallèle aux lattes. }\end{array}$} & $\begin{array}{l}\text { Exploration et } \\
\text { prise de position }\end{array}$ & $\begin{array}{l}\text { Identifier et dégager le bon } \\
\text { geste pour en faire un modèle }\end{array}$ \\
\hline & & Exécution & $\begin{array}{l}\text { Franchir la haie, jambe } \\
\text { d'esquive fléchie sur le côté. }\end{array}$ \\
\hline & \multirow{2}{*}{$\begin{array}{l}\mathrm{N}^{\circ} 3: \text { Faire quatre } \\
\text { foulées dans chaque } \\
\text { espace inter haies }\end{array}$} & $\begin{array}{l}\text { Exploration et } \\
\text { prise de position }\end{array}$ & $\begin{array}{l}\text { Repérer et dégager le bon geste } \\
\text { pour en faire un modèle. }\end{array}$ \\
\hline & & Exécution & $\begin{array}{l}\text { Adapter l'amplitude de ses } \\
\text { foulées pour ne pas piétiner } \\
\text { entre les haies }\end{array}$ \\
\hline
\end{tabular}

Tableau 5 : Synopsis condensé de la troisième séance déroulée par $E_{1}$.

\begin{tabular}{|c|c|c|c|}
\hline Contenus & Tâches & Capacités & Objectifs visés pour la séance \\
\hline \multirow{4}{*}{$\begin{array}{l}\text { Franchissement } \\
\text { de la haie et } \\
\text { reprise de la } \\
\text { course }\end{array}$} & \multirow{2}{*}{$\begin{array}{l}\mathrm{N}^{\circ} 1 \text { : Franchir les haies } \\
\text { jambe d'attaque tendue } \\
\text { en écartant sur le côté le } \\
\text { genou de la jambe libre. }\end{array}$} & $\begin{array}{l}\text { Exploration } \\
\text { et prise de } \\
\text { position }\end{array}$ & $\begin{array}{l}\text { Identifier et distinguer le rôle de } \\
\text { chaque jambe. }\end{array}$ \\
\hline & & Exécution & $\begin{array}{l}\text { Conserver le rôle de chaque } \\
\text { jambe dans le franchissement. }\end{array}$ \\
\hline & \multirow{2}{*}{$\begin{array}{ll}\mathrm{N}^{\circ} 2: & \text { Reprendre } \\
\text { activement la course } \\
\text { sans perte de vitesse. }\end{array}$} & $\begin{array}{l}\text { Exploration } \\
\text { et prise de } \\
\text { position }\end{array}$ & $\begin{array}{l}\text { Identifier le rôle des bras dans } \\
\text { l'action de franchissement. }\end{array}$ \\
\hline & & Exécution & $\begin{array}{l}\text { Franchir avec synchronisation } \\
\text { des bras pour s'équilibrer et } \\
\text { enchainer la course au } \\
\text { franchissement }\end{array}$ \\
\hline
\end{tabular}

De l'analyse du tableau 3, il ressort que le savoir enseigné par $\mathrm{E}_{1}$ à la première séance est relatif au repérage du pied d'appel et non au franchissement de la haie tel qu'il l'a annoncé avant de commencer la séance. Ce faisant, il fait montre d'un rapport très peu favorable à l'enseignement de la course de haies car ce savoir est inexistant, mais aussi confirme les assertions de Leutenegger $(2009$; 2003) selon lesquelles, les dires des enseignants peuvent ne pas être en conformité avec les actions réellement menées au contact des élèves. Toutefois, $\mathrm{E}_{1}$ en proposant pour la première séance un contenu de formation différent de celui prévu par les documents guide et programme (franchissement de la haie), semble opérer une transposition didactique interne. Ce qui semble contraire à la logique interne de l'APS vu le contenu abordé (repérage du pied d'appel). Néanmoins, contrairement aux résultats des travaux d'Oguéboulé (2008) selon lesquels, les enseignants intervenant selon l'APC abordent la première séance sans objet d'apprentissage sur la 
pratique de l'APS, E 1 est rentré directement dans la pratique avec ses élèves dès la première séance. A ce propos, il soutient à l'entretien post-séance "qu'il vaut mieux ne pas attendre la deuxième séance avant de rentrer dans la pratique de l'APS, surtout que les trois séances ne suffisent pas pour transformer la motricité de l'élèves ».

L'analyse du tableau 4 permet d'affirmer que conformément à ses propos: "l'élève apprendra à franchir les haies », $\mathrm{E}_{1}$ a enseigné le franchissement de la haie au cours de la deuxième séance. Les tâches qu'il a proposées mettent les élèves dans une position qui leur permet de s'exercer à franchir la haie. Il a même abordé l'enseignement de la course inter-haies. Seulement, en imposant un nombre de foulées entre les haies à tous les élèves qui n'ont pas les mêmes longueurs de foulées, il est possible que bon nombre d'entre eux n'arrivent pas à faire le même nombre de foulées exigé; donc à bien jouer le rôle que leur confère leur positon d'élèves dans l'institution qu'est la classe. Le rapport personnel de $E_{1}$ à l'APS course de haies peut permettre d'expliquer la progression pédagogique qu'il propose. D'ailleurs, à la fin de la séance, il a reconnu que «la majorité des élèves n'a pas réussi le franchissement et la course inter-haies ». D'autres contenus de savoirs peuvent avoir fait l'objet de la troisième séance.

En analysant tableau 5, on peut déduire que $\mathrm{E}_{1}$ a effectivement, par le biais de l'exercice répétitif permis aux élèves de faire la course de haies en plusieurs séries. Convaincu de la moindre réussite de ses élèves, il est revenu sur la course inter-haies, le franchissement et la reprise de la course après franchissement. Ses propos à la fin de la troisième et dernière séance sont en parfaite harmonie avec ceux des deux premières séances.

On peut retenir que $E_{1}$ a fini le cycle de la course de haies avec ses élèves en reconnaissant que leurs acquis sont loin d'être ceux visés par les documents officiels et prévus par lui-même. Les élèves sont donc, lors de l'apprentissage de cette APS, certainement confrontés à des difficultés qu'il faut élucider.

Au nombre des enseignants investis au CEG Okédama, il y a celui qui a en charge la $4{ }^{\text {ème }} \mathrm{C}$. Il s'agit de $\mathrm{E}_{2}$ dont les séances sont aussi analysées et interprétées.

\subsubsection{Les savoirs mis en jeu par l'enseignant $E_{2}$}

L'analyse de chacune des trois séances de cet intervenant a permis de mettre en exergue les contenus qu'il a enseignés en situation réelle de classe. Ils sont récapitulés par le biais des tableaux 6,7 et 8 . 
Tableau 6 : Synopsis condensé de la première séance déroulée par $E_{2}$.

\begin{tabular}{|c|c|c|c|}
\hline Contenus & Tâches & Capacities & Objectifs visés pour la séance \\
\hline \multirow{6}{*}{$\begin{array}{l}\text { Représentation } \\
\text { initiale de la } \\
\text { course de haies } \\
\text { et repérage du } \\
\text { pied d'appel } \\
\text { pour attaquer la } \\
1^{\text {ere haie }}\end{array}$} & \multirow{3}{*}{$\begin{array}{l}\mathrm{N}^{\circ} 1 \text { : Les élèves disent } \\
\text { et font ce qu'ils savent } \\
\text { de la course de haies }\end{array}$} & Expression & $\begin{array}{l}\text { Evaluer les acquis antérieurs des } \\
\text { élèves à la course de haies }\end{array}$ \\
\hline & & Analyse & $\begin{array}{l}\text { Faire un bilan des différentes } \\
\text { phases de la réalisation de la course } \\
\text { de haies }\end{array}$ \\
\hline & & Planification & $\begin{array}{l}\text { Etablir un ordre d'apprentissage des } \\
\text { différentes phases identifiées. }\end{array}$ \\
\hline & \multirow{3}{*}{$\begin{array}{l}\mathrm{N}^{\circ} 2 \text { Repérage du pied } \\
\text { d'appel en courant de } \\
\text { la ligne de départ à la } \\
\text { première haie. }\end{array}$} & Exploration & $\begin{array}{l}\text { Chercher par tâtonnement à repérer } \\
\text { son pied d'appel }\end{array}$ \\
\hline & & $\begin{array}{l}\text { Prise } \\
\text { position }\end{array}$ & $\begin{array}{l}\text { Identifier et dégager le bon geste } \\
\text { pour en faire un modèle } \\
\text { d'exécution }\end{array}$ \\
\hline & & Exécution & $\begin{array}{l}\text { Attaquer la première haie avec la } \\
\text { jambe libre repérée après huit } \\
\text { foulées au plus. }\end{array}$ \\
\hline
\end{tabular}

Tableau 7 : Synopsis condensé de la deuxième séance déroulée par $\mathrm{E}_{2}$

\begin{tabular}{|l|l|l|l|}
\hline Contenus & Tâches & Capacités & Objectifs visés pour la séance \\
\hline & $\begin{array}{l}\mathrm{N}^{\circ} 1: \text { Exécuter huit } \\
\text { foulées du départ à la } \\
\text { première haie. Franchir }\end{array}$ & $\begin{array}{l}\text { Exploration et } \\
\text { prise de position }\end{array}$ & $\begin{array}{l}\text { Faire huit foulées du départ à la } \\
\text { première haie Conserver la même } \\
\text { jambe d'attaque au } \\
\text { fe la haie et } \\
\text { franchissement. }\end{array}$ \\
$\begin{array}{l}\text { Course } \\
\text { rythmique dans } \\
\text { l'espace inter- } \\
\text { haies }\end{array}$ & $\begin{array}{l}\text { d'attaque tendue en } \\
\text { écartant sur le côté le } \\
\text { genou de la jambe } \\
\text { libre. }\end{array}$ & Exécution & $\begin{array}{l}\text { Faire passer le genou de la jambe } \\
\text { libre sur le côté. }\end{array}$ \\
\cline { 2 - 4 } & $\begin{array}{l}\mathrm{N}^{\circ} 2: \text { Faire au plus } \\
\text { quatre foulées dans } \\
\text { chaque espace inter } \\
\text { haies }\end{array}$ & $\begin{array}{l}\text { Exploration et } \\
\text { prise de position }\end{array}$ & $\begin{array}{l}\text { Exécution } \\
\text { pour en faire un modèle. }\end{array}$ \\
\hline
\end{tabular}

Tableau 8: Synopsis condensé de la troisième séance déroulée par $\mathrm{E}_{2}$

\begin{tabular}{|l|l|l|l|}
\hline Contenus & Tâches & Capacités & Objectifs visés pour la séance \\
\hline & $\begin{array}{l}\mathrm{N}^{\circ} 1: \\
\text { correctement course et } \\
\text { impulsion pour mieux } \\
\text { franchir les haies. }\end{array}$ & $\begin{array}{l}\text { Exploration et } \\
\text { prise de position }\end{array}$ & $\begin{array}{l}\text { Identifier le bon geste pour en } \\
\text { faire un modèle. }\end{array}$ \\
\cline { 2 - 4 } $\begin{array}{l}\text { Liaison course } \\
\text { franchissement } \\
\text { et la reprise de } \\
\text { course }\end{array}$ & \begin{tabular}{l}
$\mathrm{N}^{\circ} 2: \begin{array}{l}\text { Reprendre } \\
\text { activement la course } \\
\text { sans perdre sa vitesse. }\end{array}$ \\
\cline { 2 - 4 }
\end{tabular} & $\begin{array}{l}\text { Exploration et } \\
\text { prise de position }\end{array}$ & $\begin{array}{l}\text { Amener à une impulsion loin de la } \\
\text { haie. }\end{array}$ \\
\cline { 2 - 4 } & l'action de franchissement. le rôle des bras dans \\
\hline
\end{tabular}

En référence à l'analyse du synopsis relatif au tableau 6 , on peut constater que, l'enseignant $\mathrm{E}_{2}$ après avoir diagnostiqué les acquis antérieurs 
de ses élèves s'inscrit dans la même logique que E1 en faisant un mauvais traitement didactique. Les mêmes raisonnements menés précédemment restent valables car il semble que $\mathrm{E}_{2}$ aussi manque d'ingrédients didactiques en terme de progression pédagogique. Les confusions entre l'atteinte ou non de ses objectifs, ce qu'il a enseigné et ce qu'il dit avoir enseigné reflètent qu'il a des rapports institutionnel et personnel peu favorable aux enjeux de savoir liés à l'APS course de haies. Il affirme à l'entretien post-séance : "Je ne pas dire que mon objectif est atteint, mais les élèves ont appris quelque chose ». Cette situation peut être l'une des sources de difficultés éventuellement rencontrées par les élèves. Les actions de $E_{2}$ ont continué avec l'étude de sa deuxième séance.

L'analyse du tableau 7 montre qu'E2 a enseigné le franchissement et la course inter-haies. La progression pédagogique suivie par cet enseignant part du nombre de foulées avant l'attaque de la première haie, passe par le franchissement pour aboutir à la course inter-haies. Par contre, les documents guide et programme prévoient chronologiquement l'enseignement du franchissement, de la course inter-haies et du nombre de foulées avant l'attaque de la première haie. Il y a donc une transposition didactique qui sous-tend un écart entre le prescrit et réalisé. Le cycle de l'enseignement de la course de haies par $\mathrm{E}_{2} \mathrm{~s}$ 'est achevé à la troisième séance.

En analysant le tableau 8, il convient de retenir qu'E2 s'est intéressé d'une part à la liaison course-franchissement puis d'autre part, à la reprise de la course après chaque franchissement. Ce faisant, il aborde l'un des aspects de l'enseignement de la course de haies évoqué par Lamotte (2002). Selon cet auteur le problème majeur rencontré par les élèves à la course de haies est la liaison permanente entre la course et le franchissement des haies. Les propos d' $\mathrm{E}_{2}$ à la fin de la dernière séance du cycle et la visualisation des séances filmées confirment les mêmes problèmes chez les élèves d'E2. Il reconnaît à la fin de la dernière séance que "les élèves n'arrivent pas à reprendre la course après le passage sur les haies ».

En recherchant toujours à explorer toutes les données recueillies de la pratique enseignante afin de mettre en exergue les difficultés rencontrées par les élèves, nous avons analysé et interprété les trois séances déroulées par E3.

\subsubsection{Les savoirs mis à l'étude par l'enseignant $E_{3}$}

L'analyse de chacune des trois séances de cet enseignant a permis d'explorer les contenus qu'il a réellement enseignés à partir des tableaux 9, 10 et 11. 
Tableau 9 : Synopsis condensé de la première séance déroulée par $E_{3}$

\begin{tabular}{|c|c|c|c|}
\hline Contenus & Tâches & Capacités & Objectifs visés pour la séance \\
\hline \multirow{6}{*}{$\begin{array}{l}\text { Franchir des } \\
\text { obstacles bas } \\
\text { et limiter } \\
\text { l'élévation } \\
\text { du centre de } \\
\text { gravité }\end{array}$} & \multirow{3}{*}{$\begin{array}{l}\mathrm{N}^{\circ} 1 \text { : Courir sur } \\
\text { obstacles bas sans } \\
\text { s'élever en } \\
\text { recherchant la notion } \\
\text { de course de vitesse }\end{array}$} & Expression & $\begin{array}{l}\text { Evaluer les acquis antérieurs des élèves } \\
\text { à la course de haies }\end{array}$ \\
\hline & & Analyse & $\begin{array}{l}\text { Identifier les différentes phases à retenir } \\
\text { pour pratiquer la course de haies }\end{array}$ \\
\hline & & Planification & $\begin{array}{l}\text { Courir et maintenir sa vitesse pour } \\
\text { franchir des haies basses }\end{array}$ \\
\hline & \multirow{3}{*}{$\begin{array}{l}\mathrm{N}^{\circ} 2 \text { : Lier course et } \\
\text { franchissement du } \\
\text { départ à la première } \\
\text { haie. }\end{array}$} & Exploration & $\begin{array}{l}\text { Ressortir les différentes étapes liées } \\
\text { franchissement de la première haie : } \\
\text { Départ-Course de vitesse-Liaison } \\
\text { course-franchissement }\end{array}$ \\
\hline & & $\begin{array}{l}\text { Prise de } \\
\text { position }\end{array}$ & $\begin{array}{l}\text { Identifier et dégager le bon geste pour } \\
\text { en faire un modèle d'exécution }\end{array}$ \\
\hline & & Exécution & $\begin{array}{l}\text { Ne pas ralentir à l'approche de la } \\
\text { première haie. }\end{array}$ \\
\hline
\end{tabular}

Tableau 10: Synopsis condensé de la deuxième séance déroulée par $\mathrm{E}_{3}$

\begin{tabular}{|c|c|c|c|}
\hline Contenus & Tâches & Capacités & Objectifs visés pour la séance \\
\hline \multirow{4}{*}{$\begin{array}{l}\text { Franchisseme } \\
\text { nt de haies sur } \\
\text { une course } \\
\text { rythmée }\end{array}$} & \multirow{2}{*}{$\begin{array}{l}\mathrm{N}^{\circ} 1 \text { : Course haute et } \\
\text { progressivement } \\
\text { accélérée pour franchir } \\
\text { les obstacles sans } \\
\text { hésiter, sans sauter. }\end{array}$} & $\begin{array}{l}\text { Exploration et } \\
\text { prise de position }\end{array}$ & $\begin{array}{l}\text { Identifier et Dégager le bon geste } \\
\text { pour en faire un modèle }\end{array}$ \\
\hline & & Exécution & $\begin{array}{l}\text { Attaquer la haie, jambe d'attaque } \\
\text { tendue et jambe d'esquive fléchie sur } \\
\text { le côté sans casser le rythme de la } \\
\text { course. }\end{array}$ \\
\hline & \multirow{2}{*}{$\begin{array}{l}\mathrm{N}^{\circ} 2 \text { : Faire une course } \\
\text { progressivement } \\
\text { accélérée entre les } \\
\text { haies. }\end{array}$} & $\begin{array}{l}\text { tion et } \\
\text { position }\end{array}$ & $\begin{array}{l}\text { Identifier et dégager le bon geste } \\
\text { pour en faire un modèle }\end{array}$ \\
\hline & & Exécution & $\begin{array}{l}\text { Faire quatre appuis inter-obstacles en } \\
\text { respectant rythme et équilibre dans le } \\
\text { franchissement }\end{array}$ \\
\hline
\end{tabular}

Tableau 11 : Synopsis condensé de la troisième séance déroulée par $\mathrm{E}_{3}$

\begin{tabular}{|c|c|c|c|}
\hline Contenus & Tâches & Capacités & Objectifs visés pour la séance \\
\hline \multirow{4}{*}{$\begin{array}{l}\text { Reprise } \\
\text { d'appuis et } \\
\text { franchisse } \\
\text { ment de la } \\
\text { ligne } \\
\text { d'arrivée. }\end{array}$} & \multirow{2}{*}{$\begin{array}{l}\mathrm{N}^{\circ} 1: \quad \text { Reprendre } \\
\text { activement la course sans } \\
\text { perte de vitesse. }\end{array}$} & $\begin{array}{l}\text { Exploration et prise } \\
\text { de position }\end{array}$ & $\begin{array}{l}\text { Identifier et distinguer le rôle de } \\
\text { chaque jambe. }\end{array}$ \\
\hline & & Exécution & $\begin{array}{l}\text { Faire un retour actif de la jambe } \\
\text { d'esquive pour se relancer dans la } \\
\text { course après } \\
\text { franchissement. }\end{array}$ \\
\hline & \multirow{2}{*}{$\begin{array}{l}\mathrm{N}^{\circ} 2 \text { : Maintenir sa vitesse } \\
\text { de course jusqu'à franchir } \\
\text { la ligne d'arrivée. }\end{array}$} & $\begin{array}{l}\text { Exploration et prise } \\
\text { de position }\end{array}$ & $\begin{array}{l}\text { Identifier le rôle des bras dans } \\
\text { l'action de franchissement. }\end{array}$ \\
\hline & & Exécution & $\begin{array}{l}\text { Courir en toute vitesse pour } \\
\text { franchir la ligne d'arrivée. }\end{array}$ \\
\hline
\end{tabular}

Il ressort de l'analyse du tableau 9 que $\mathrm{E}_{3}$ dans un premier temps a vérifié les pré-requis de ses élèves par le franchissement d'obstacles bas en vitesse puis dans un second temps, il a enseigné la liaison coursefranchissement de la première haie en vitesse. Il est ainsi en harmonie avec 
Lamote (2002) qui affirme que la logique du cycle est de maîtriser la liaison élan/impulsion afin de réaliser un saut en longueur dans lequel on distingue bien l'élan, l'impulsion, l'envol et la réception. Pour ce contenu enseigné qui est déjà une étape de la course de haies, $\mathrm{E}_{3}$ a encore fait des régulations visant à aider ses élèves à identifier des sous-étapes, certainement pour leur faciliter l'apprentissage. Ce faisant, il opère une transposition interne sur le savoir à enseigner compte tenu de sa posture topogénique dans l'institution classe et de rapport à l'enseignement de la course de haies. Qu'a t'il enseigné alors lors de sa deuxième séance ?

On peut retenir du tableau 10 que, $\mathrm{E}_{3}$ a déroulé sa séance en enseignant comme contenus de savoirs, le franchissement des haies et la course rythmique inter-haies. Dans cette logique, E3 a respecté l'ordre prévu par les documents officiels pour l'enseignement des objets même si des restrictions ont été opérées au niveau des techniques et connaissances prescrites par le guide et le programme.

A la troisième et dernière séance du cycle, après avoir analysé le tableau 11, Il convient de retenir que $\mathrm{E}_{3}$ a enseigné la reprise active de la course sans perte de vitesse après le franchissement (Lamotte, 2002) et le maintien de cette vitesse jusqu'à la ligne d'arrivée. Il a avoué lui-même au cours de l'entretien post-séance que "les élèves n'ont pas pu réaliser le bon geste du franchissement des haies ». Cette insuffisance dans l'apprentissage des élèves constitue alors une source probable des difficultés évoquées en fin de cycle. Dans la suite de nos investigations, nous avons élucidé et expliqué les difficultés rencontrées par les élèves face aux tâches proposées par les enseignants.

\subsection{De l'intérêt d'une approche didactique centrée sur les praxéologies didactiques et disciplinaires à la course de haies}

Pendant les différents entretiens, $\mathrm{E}_{1}, \mathrm{E}_{2}$ et $\mathrm{E}_{3}$ ont mis l'accent sur les difficultés des élèves exécutant les tâches à eux proposées. Les élèves interrogés à l'entretien post-cycle ont eu aussi l'occasion d'évoquer les difficultés qu'ils ont rencontrées lors de l'apprentissage de la course de haies. Les informations issues de ces entretiens ont été confrontées dans la logique de la triangulation (Leutenegger, 2009 ; Van Der Maren, 1996) à celles recueillies par observation in-situ des interactions didactiques. Cette mise en tension nous a permis d'inférer dans un tableau, les difficultés rencontrées par les élèves lors de l'initiation à la course de haies. 
Tableau 12 : Récapitulation des difficultés rencontrées par les élèves lors de l'apprentissage des objets enseigné par $\mathrm{E}_{1}, \mathrm{E}_{2}$ et $\mathrm{E}_{3}$.

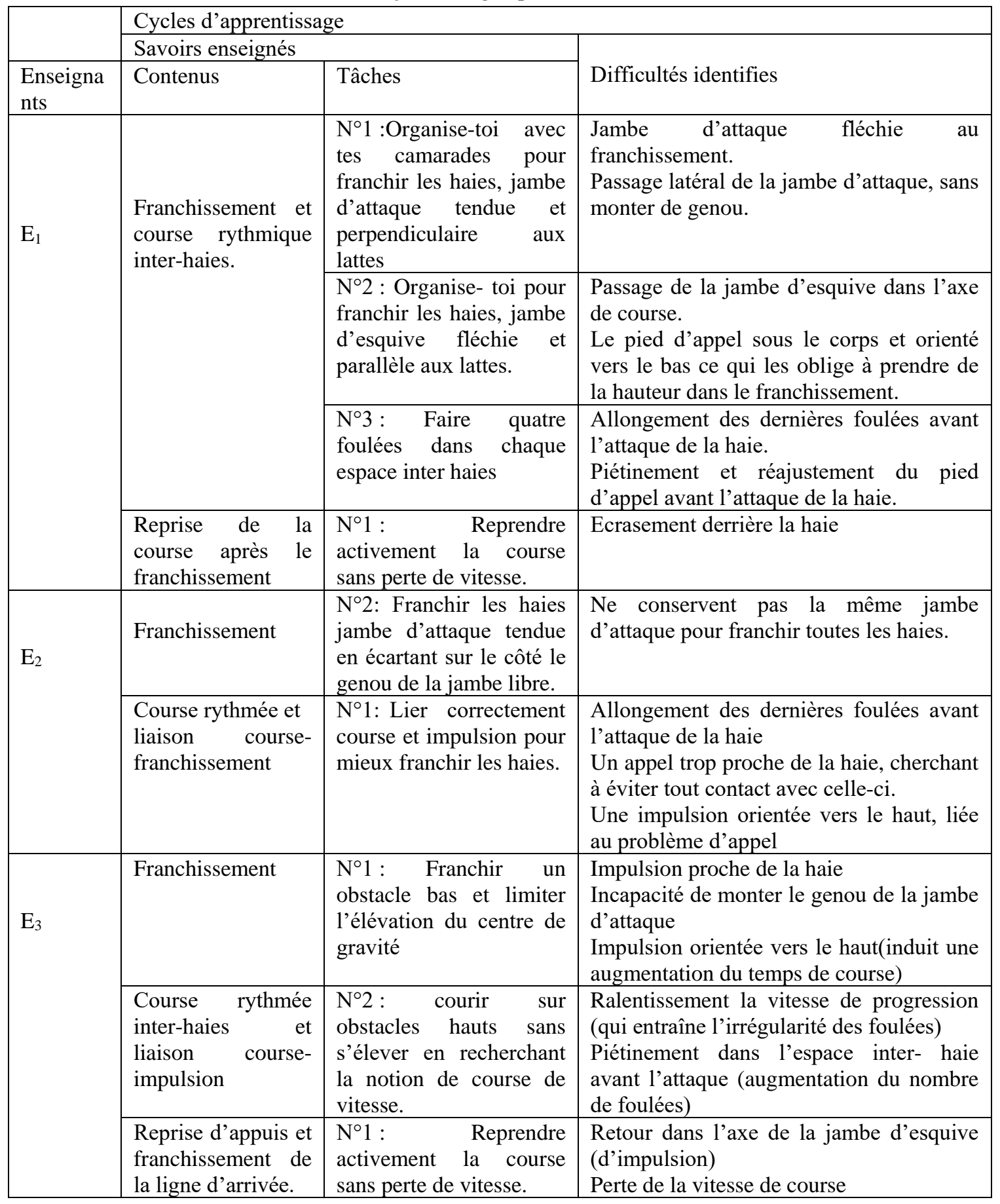


De l'analyse de ce tableau, il se dégage trois niveaux de difficultés : le franchissement, la course inter-haies et la reprise de course après le franchissement.

\subsubsection{Le franchissement}

D'abord pour attaquer la haie, l'appel a lieu très proche de celle-ci et contraint à une suspension orientée beaucoup plus vers le haut que vers l'avant. Cette difficulté des élèves du CEG Okédama est en harmonie avec celles évoquées par Blondel et Chevallier (2010), Lamotte (2002). Ces auteurs parlent d'attaque trop près de la haie, du saut de la haie et de l'élévation du centre de gravité des élèves lors du franchissement. Ensuite, la jambe d'attaque n'est pas constante. Elle change d'un franchissement à l'autre et reste fléchie au lieu d'être tendue. Parfois, cette jambe ne passe pas dans l'axe de la course lors du franchissement. C'est la situation à laquelle Lamotte (2002) fait allusion en parlant de la jambe d'attaque durant la première leçon de course de haies. Enfin, la jambe d'appel lors de l'esquive passe dans l'axe de course au lieu de passer latéralement. Le pied est sous le corps, orienté vers le bas et contraint aussi à élever le centre de gravité pour éviter l'obstacle. Il s'agit du " passage de la jambe d'appel en crochet sous l'élève » (Lamotte, 2002, p 192). Les propos des élèves à l'entretien postcycle sont en harmonie avec les réalités issues des observations des séances de classe et permettent d'affirmer que l'univers cognitif des élèves (Chevallard, 1997) est caractérisé par la présence de savoirs non conformes à ceux institutionnalisés par les enseignants. En voici quelques extrais : "Nous avons des difficultés à sauter les obstacles", "Nous changeons de pieds d'un obstacle à l'autre ", "Nous ne savons pas comment faire pour bien sauter les obstacles ». Ce sont des dires qui prouvent que les élèves ont retenu que les haies sont à sauter, alors qu'il s'agit en fait d'un franchissement et non d'un saut.

\subsubsection{La course inter-haies}

Nombreuses sont les difficultés qui émergent de la réalisation de la course inter-haies. Il s'agit du piétinement et de l'allongement pour respecter le nombre de foulées exigé. Certains élèves ralentissent carrément à l'approche de la haie, occasionnant ainsi une irrégularité de la course qui perturbe l'attaque de la haie. Les élèves interrogés enfin de cycle ont reconnu qu'ils n'arrivent pas à respecter le nombre de foulées proposés par les enseignants. Les résultats des travaux évoqués dans le cas du franchissement (Pradet et Soler, 2004 ; Dupré et Janin, 2003) sont en synergie avec ceux relevés chez les élèves lors de la course inter-haies au CEG Okédama de Parakou au Bénin. 


\subsubsection{La reprise de la course après le franchissement}

$\mathrm{Au}$ lieu d'atterrir sur la pointe des pieds, les élèves posent entièrement la plante du pied au sol après le franchissement. C'est un écrasement qui occasionne un déséquilibre, une perte de vitesse ou même un arrêt de la course. Dans cette logique, un des élèves interrogés a affirmé que " quand on met les pieds au sol après l'obstacle, on sent des douleurs et c'est comme si on veut tomber ». Cette situation peut donc être aussi source de lésion ostéologique et/ou myologique. A cet effet, Lamotte (2002) et Aubert (1994) parlent d'effondrement après obstacle qui rend la reprise de la course fastidieuse. C'est surtout à cette phase que l'enseignant doit faire attention pour que l'envie d'apprendre ne soit pas effritée au niveau de l'élève (Humbert, Le Levé et Roger, 2009).

A propos des difficultés, il faut retenir que les professeurs proposent des tâches visant l'enseignement d'un contenu. Mais force est de constater que les élèves réalisent des gestes qui ne sont pas ceux attendus par le professeur. Les difficultés engendrent des écarts entre ce que l'enseignant demande et ce que font réellement les élèves qui peuvent finir par acquérir une motricité non en conformité avec celle visée par le professeur pour la course de haies. Dans le cas de cette étude, nous avons exploré les sources éventuelles de ces difficultés. Cela a abouti, en référence à la revue de littérature, à une proposition des tâches pouvant permettre d'enseigner les mêmes contenus avec moins de difficultés.

\subsection{Les difficultés d'ordre didactiques rencontrées par les élèves : éléments de discussion}

Les propos des acteurs du système didactique révèlent que les élèves du CEG Okédama sont dans un contexte qui leur permet de découvrir et de pratiquer la course de haies seulement au collège. Dans ce cas, les prescriptions devraient rendre le premier contact plus ludique avant l'évolution vers les contraintes de technicités avancées. C'est ce que préconisent d'ailleurs Dupré et Janin (2003), Aubert (1994), Seners (1993). Les documents officiels posent donc eux-mêmes problèmes quant à ce qui concerne la progression pédagogique dans l'enseignement de la course de haies. Il s'agit selon Chevallard (1999) de la praxéologie de l'APS objet d'enseignement. Les savoirs et savoir-faire retenus par le programme sont trop nombreux pour les débutants que sont les élèves de la classe de $4^{\text {ème }}$ qui n'ont que trois séances pour apprendre une APS comme la course de haies qui a trop de contraintes physiques et techniques.

Dans ce contexte, il est normal que les enseignants éprouvent d'énormes difficultés dans le choix des objets d'enseignement à la course de haies. C'est le cas, nous l'avons constaté de $\mathrm{E}_{1}, \mathrm{E}_{2}$ et $\mathrm{E}_{3}$ qui eux-mêmes ont des rapports personnel et institutionnel peu favorables à l'objet de savoir 
qu'est la course de haies. En imposant par exemple un nombre de foulées à tous les élèves d'une classe sur une distance fixe, ils en ajoutent aux difficultés des élèves du fait de la variabilité des longueurs de foulées d'un élève à l'autre. Ils n'arrivent même pas à modifier la hauteur des haies selon les longueurs segmentaires des élèves. En tout cela ne pourrait même pas se faire vu les conditions matériels très peu reluisantes de l'apprentissage(Absence de haies adéquates, terrain très peu pratique etc.) Cette situation qui a pour corollaire, le piétinement au niveau de la course, rend difficile le franchissement et l'atterrissage après l'obstacle. Dans ce cas, la reprise de la course devient périlleuse. Les élèves ne savent même pas qu'il faut atterrir sur la pointe du pied afin d'éviter l'écrasement.

Lors de l'enseignement des différentes techniques relatives à la course de haies, les propos des élèves à l'entretien post-cycle confirmés par les visualisations des séquences de classe ont révélé que les enseignants n'interviennent pas auprès des élèves pour leur apporter des solutions aux problèmes auxquels ils sont confrontés. Il ne peut en être autrement dans un contexte où la mise en œuvre de l'enseignement des APS selon l'APC prescrit aux enseignants un comportement qui les amène à se contenter de faire découvrir les APS et à rester dans la globalité avec plus d'importance à la démarche qu'à la pratique par les élèves (Agbodjogbé, 2013; 2007 ; Oguéboulé, 2008 ; Atoun, 2005). D'ailleurs, les trois séances réservées à l'enseignement de la course de haies ne peuvent pas suffire pour espérer un changement moteur chez des élèves qui non seulement découvrent l'APS pour la première fois, mais aussi n'ont aucune autre occasion de la pratiquer. Nous savons en référence aux travaux de Delignères (1994) que, pour modifier une technique, il faut de nombreuses répétitions et que la course de haies est un sport de base qui est très technique (Terret, 2002 ; Goriot, 1980). Pour amoindrir ces difficultés, Pradet et Soler (2004), Dupré et Janin (2003), Lamote (2002), Aubert (1994), Seners (1993) et le Cercle d'Etudes Athlétisme des ENSEPS (1967) avaient déjà fait des propositions. Elles sont applicables aux élèves béninois qui, pour le cas du CEG Okédama à Parakou ont les mêmes difficultés.

\section{Conclusion}

La présente étude d'obédience didactique a été initiée pour mettre en évidence les difficultés rencontrées par les élèves lors de l'enseignement apprentissage à la course de haies en $4^{\mathrm{e}}$. Elle a été conduite à l'aide d'une démarche inspirée par des concepts mobilisés à partir de la théorie de l'action didactique conjointe (Sensevy, 2007; Amade-Escot, 2003) et la théorie de l'anthropologique du didactique (Chevallard, 1999 ; 1997 ; 1992 ; Verret, 1975) déjà évoquées. Les résultats, analysés et interprétés confirment 
la majorité des travaux précédents et notre hypothèse. Ils permettent aussi de faciliter l'enseignement/apprentissage de la course de haies au Bénin.

Les enseignants investis adaptent de façon générique, les prescriptions du programme aux réalités de leur milieu d'intervention en opérant des choix transpositifs, en synergie avec les travaux des résultats de Oguéboulé, Agbodjogbé, Attiklémè, Kouété et Kpazaï (2016) sur le lancer de javelot. Dans cette logique, l'ordre de proposition des activités d'apprentissage prescrit n'est pas toujours respecté. D'ailleurs, les contenus de savoirs au programme constituent l'une des causes des difficultés des élèves en apprentissage de la course de haies. Ils sont trop nombreux, listés sans aucune chronologie et correspondent beaucoup plus aux athlètes confirmés qu'aux élèves de $4^{\mathrm{e}}$, découvrant l'APS et n'ayant que trois séances fortement théorisées comme le disaient déjà Agbodjogbé $(2013$; 2007), Agbotouédo (2008), Oguéboulé (2008), Atoun (2005). Le rapport personnel des élèves à la course de haies rend donc difficile l'enseignement et surtout l'apprentissage.

Conformément à notre hypothèse, les élèves ont eu des difficultés à apprendre les savoirs à eux proposés par les enseignants. Trois difficultés majeures émergent du comportement des élèves en situation ordinaire de classe. Elles sont relatives aux objets d'enseignement que sont: le franchissement, la course inter-haies et la reprise de la course après le franchissement. Face aux difficultés des élèves, $E_{1}, E_{2}$ et $E_{3}$ n'arrivent pas à intervenir pour insister sur les opérations motrices attendues et attirer l'attention sur ce qu'il ne faut pas faire. Ils se contentent de les faire passer et repasser sans poser l'acte qui permet d'avoir le changement moteur escompté. Leur rapport personnel et institutionnel à l'objet de savoir qu'est la course de haies permet d'expliquer cette situation. De plus, la foultitude de connaissances et techniques à enseigner et la méconnaissance de l'APS les mettent dans un embarras de choix. En trois séances, il est difficile, voire impossible d'espérer un changement moteur chez des élèves qui sont à leur premier contact avec la course de haies.

\section{References:}

1. Abaya, V. (2004). Problématique de l'enseignement des sports collectifs dans les collèges et lycées du Bénin : cas du football dans la ville de Porto-Novo. Mémoire de Maîtrise en STAPS, PortoNovo : INJEPS-UAC. 52p.

2. Agassounon, R. (2013). Problématique de l'enseignement du basketball selon l'approche par les compétences dans les classes de

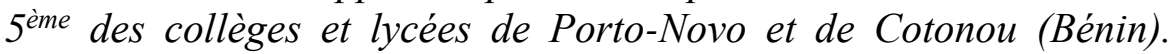
Mémoire de Maîtrise en STAPS, Porto-Novo : INJEPS-UAC. 77p. 
3. Agbodjogbé, D.B. (2013). L'implémentation des nouveaux programmes par compétences au Bénin : des textes officiels aux pratiques d'enseignement. Analyses didactiques en Education Physique et Sportive et en Science de la Vie et de la terre en classe de 5ème. Thèse de Doctorat publiée, Université Toulouse 2 Le Mirail (UT2 Le Mirail). 540p.

4. Agbodjogbé, D.B. (2007). Problématique de la mise en cuvre de la démarche d'enseignement /apprentissage/évaluation en EPS selon les programmes par compétences au Bénin. Mémoire de Master II Recherche en éducation physique, sport et développement humain, Porto-Novo : INJEPS-UAC. 81p.

5. Agbotouédo, P. A. (2008). Problématique de l'enseignement de la course de vitesse selon l'approche par compétences au Bénin. , Porto-Novo : Mémoire de Maîtrise en STAPS, Porto-Novo : INJEPSUAC.50p.

6. Amade-Escot, C. (2008). Recherches didactiques en situation d'intervention dans les APSA ; concepts et Méthodes. Enseignement pour les étudiants en Master II. Porto-Novo : Recherche en STAPS, l'INJEPS-UAC.

7. Amade-Escot, C. (2007). Les savoirs au cour du didactique. In C. Amade-Escot, (coord), le didactique. Paris : Edition Revue EPS, collection « Pour l'action », pp 11-30.

8. Amade-Escot, C. (2003). La gestion interactive du contrat didactique en volleyball : agencement des milieux et régulations du professeur. In C. Amade-Escot (Ed.), Didactique de l'éducation physique Etat des recherches (pp.240-264). Paris : Editions de la revue EPS.

9. Amade-Escot, C., Garnier, A., \& Monnier, N. (2007). La dynamique contractuelle du processus didactique. In Chantal Amade-Escot (direction), Le didactique (pp.31-48). Paris : Edition Revue EPS.

10. Atoun, E. C. (2005). Les nouveaux programmes d'EPS au Bénin : une approche didactique des contenus définis en termes de compétences. Mémoire de Maîtrise en STAPS, Porto-Novo: INJEPS-UAC. 78p.

11. Atoun, C., Agbodjogbé, B., Attiklémè, K., Oguéboulé, B.M. \& Kpazaï, G. (2015). L'évaluation en sports collectifs selon l'APC dans les collèges de l'Ouémé : le cas de l'enseignement du volleyball. La recherche en éducation, 13, 48-65.

12. Attiklémè, K. (2009). L'analyse comparée des savoirs enseignés en natation dans les classes de $6^{\text {ème }}$ des collèges et lycées du Bénin et de la France. Laboratoire des Sciences de l'Homme et de la Société de l'INJEPS/UAC (Bénin) et le DiDiST-CREF-T de l'Université de Toulouse (France), pp 41-67. 
13. Attiklémè, K. \& Kpazaï, G. (2011). Analyse de l'évolution des curricula de l'enseignement de l'éducation physique et sportive (EPS) en République du Bénin de la période coloniale à nos jours. Recherche en Education, $\mathrm{n}^{\circ} 164$, pp 83-99.

14. Aubert, F. (1994). La course de haies haute. Revue EPS N²46.

15. Bankolé, E. (2013). Adaptation des contenus de programme par compétences en EPS au contexte d'apprentissage à la course de haies au CEG Agbokou de Porto-Novo au Bénin. Mémoire de Master1 STAPS, Porto-Novo : INJEPS-UAC. 64p.

16. Blondel, P. \& Chevalier, B. (2010). Courses de haies : courir vite en franchissant des obstacles. Revue EPS. Cahier 12 ans et plus. $\mathrm{N}^{\circ} 343$.

17. Boni, J. P. (2011). L'analyse des interactions didactiques de trois enseignants stagiaires de la Licence-STAPS : le cas de la gymnastique. Mémoire de Maîtrise en STAPS; Porto-Novo : INJEPSUAC. 70p.

18. Cercle d'études d'Athlétisme de l'ENSEPS. (1967). La pratique d'athlétisme. Supplément au No88 de la Revue EPS, Septembre.

19. Chevallard, Y. (1999). L'analyse des pratiques enseignantes en théorie anthropologique du didactique. Recherche en didactique des mathématiques, 19 (2). pp 221-266.

20. Chevallard, Y. (1997). Les savoirs enseignés et leurs formes scolaires de transmission : un point de vue didactique. Sklolé, 7, $\mathrm{p} 101$.

21. Chevallard, Y. (1992). Concepts fondamentaux de la didactique : perspectives apportées par une approche anthropologique. Recherche en didactique de mathématique, Marseille, Université Aix-Marseille II. Vol. 12, $N^{\circ} 1$, pp 73-112.

22. Chevallard, Y. (1991). Didactique, anthropologie, mathématique. In

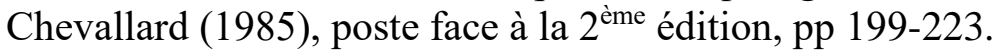

23. Delignères, D. (1994). Education physique et santé. Cours d'agrégation externe EPS, Ecrit 2 Centre National d'Enseignement à distance.

24. Devos-Prieur, O. (2006). Rapports aux savoirs des professeurs d'école et développement des contenus en éducation physique. Etude comparée de quatre cycles de basket-ball au cours moyen. Thèse de Doctorat de sciences de l'éducation non publiée, Toulouse: Université Paul Sabatier.

25. Direction de l'Enseignement Secondaire Général. (2014). Recueil de données statistiques des collèges et lycées publics de l'enseignement secondaire général. Porto-Novo: Service de l'organisation scolaire et de la prévision.

26. Direction de l'Enseignement Secondaire Général. (2015). Recueil de données statistiques des collèges et lycées publics de l'enseignement 
secondaire général. Porto-Novo : Service de l'organisation scolaire et de la prévision.

27. Dupré, R. \& Janin, D. (2003). Enseigner les courses de relais et de haies en milieu scolaire (Collèges et lycées).Editions ACTIO.

28. Fagnon, V.C.R.S. (2010). Etude comparative au saut en longueur dans le département de l'Ouémé- Plateau. Mémoire de Maîtrise en STAPS. Porto-Novo : INJEPS-UAC, 60p.

29. Gangbadja, C. M. (2013). Problématique de l'enseignement de la course de relais selon l'approche par compétences dans les lycées et collèges de l'Ouémé-Plateau. Mémoire de Master-1-STAPS, PortoNovo : INJEPS-UAC. 62p.

30. Goriot, G. (1980). Les fondamentaux de l'athlétisme. Paris : Vigot.

31. Hubiche, J. L. \& Pradet, M. (2000). Comprendre l'athlétisme. Paris : INSEP-Publication.

32. Humbert, Y., Le Levé, E. \& Roger, A. (2009). Athlétisme : course de haies. WWW.contrepiednet.

33. Jeannin, M. (2011). Programme EPS, compétences attendues dans les APSA et socle commun de connaissances et de compétences. France : Créteil.

34. Jonnaert, Ph. (2002). Compétences et socioconstructivisme. Un cadre théorique. Perspectives et Education en Formation. Bruxelles: Edition De Boeck.

35. Lamotte, V. (2002). Leçon d'EPS : Discours et Réalité. Dossier EPS $\mathrm{N}^{\circ} 47,319 \mathrm{p}$.

36. Legendre, R. (1993). Le dictionnaire actuel de l'éducation. Paris : Edition ESKA.

37. Leutenegger, F. (2009). Le temps d'instruire. Approche clinique et expérimentale $d u$ didactique ordinaire en mathématiques. Berne, Peter Lang.

38. Leutenegger, F. (2003). Etude des interactions didactique en classe de mathématiques: un prototype méthodologique. Bulletin de psychologie vol 56, $\mathrm{N}^{\circ} 4$ pp 559-571.

39. Marsenach, J. \& Mérand, R. (1987). L'évaluation formative en Education Physique et Sportive dans les collèges. Rapport Scientifique-INRP Marsenach, J. (1991). EPS : quel enseignement? Paris : INRP. 33p.

40. Martinand, J. L. (1989). Pratique de référence, transposition didactique et savoir professionnel en sciences et techniques. Les sciences de l'éducation, 2, pp 23-29.

41. Mercier, A., Schubauer-Leoni, M-L. \& Sensevy, G. (2002). Vers une didactique comparée. Revue française de pédagogie, $N^{\circ} 141$ OctobreNovembre-Décembre, pp 5-16. 
42. Ministère de l'enseignement Secondaire, de la Formation Technique et Professionnelle, de la Reconversion et de l'Insertion des Jeunes. (2015). Guide pédagogique en EPS classe de $4^{\mathrm{ème}}$. Inspection Générale Pédagogique du Ministère; Porto-Novo, Direction de l'Enseignement Secondaire.

43. Ministère de l'Enseignement Secondaire, de la Formation Technique et Professionnelle, de la Reconversion et de l'Insertion des Jeunes. (2015). Programme d'études en EPS classe de $4^{\text {ème }}$. Inspection générale pédagogique du Ministère; Porto-Novo, Direction de l'enseignement secondaire.

44. Ministères de l'Education Nationale. (2007). Forum national sur le secteur de l'éducation: les programme d'études, approches méthodologique, enjeu et perspectives. Cotonou.

45. Odjousou, M. (2010). La structuration de l'enseignement de l'éducation physique et sportive au Bénin à partir d'un nouveau programme : enjeu d'une mutation ou mutation d'un enjeu. Thèse de Doctorat en STAPS. Mention Didactique, Expertise, et Technologie en APS Science Humaine et Sociale. Renne: sous le sceau de l'Université Européenne de Bretagne, 312p.

46. Oguéboulé, B. M. (2017). La problématique de la différence de sexe lors du processus enseignement /apprentissage en EPS : Un regard didactique de l'enseignement $d u$ volleyball au secondaire. Thèse de Doctorat en STAPS. Ecole Doctorale Pluridisciplinaire : Education Physique, Sport et Développement Humain. Spécialité Didactique des APS. INJEPS-UAC, Porto-Novo. 285p.

47. Oguéboulé, B. M. (2008). Les effets emblématiques du nouveau programme d'EPS au Bénin : le cas de la $S A N^{\circ} 1$ dans les classes de $6^{\text {ème }}$ des établissements secondaires de l'Ouémé (Le Lycée Toffa $1^{\text {er }}$ et le CEG1Adjara). Mémoire de Master 2 Recherche en STAPS, PortoNovo : INJEPS-UAC. 45p.

48. Oguéboulé, B.M, Agbodjogbé, D.B., Attiklémè, K., Kouété, C. \& Kpazaï, G. (2016). Adaptation des contenus du programme par compétences en EPS au contexte d'apprentissage : une lecture de trois cas de l'enseignement du javelot au Bénin. La revue universitaire des sciences de l'éducation, assempe: Editions Universitaires de la Côte d'Ivoire. $\mathrm{N}^{\circ} 6$, pp 58-81.

49. Perrenoud, P. (1998). La transposition didactique à partir de pratiques : des savoirs aux compétences. Faculté de psychologie et des sciences de l'éducation, Genève : Université de Genève.

50. Poussin, B. (2009). Observation didactique d'un maître en formation lors d'une séquence d'enseignement innovante en jeux sportifs 
collectifs. Institut de formation des maîtres et maîtresses de l'enseignement secondaire, Genève: eJRIEPS $N^{\circ} 18$, pp 85-106.

51. Pradet, M. \& Soler, A. (2004). La course de haies en situation. L'EPS en poche, Paris : Revue EPS.

52. Seners, P. (1993). La leçon d'EPS. Paris : Vigot.

53. Sensevy, G. (2007). Des catégories pour décrire et comprendre l'action conjointe. In G. Sensevy et A. Mercier (Eds.). Agir ensemble, l'action didactique conjointe du professeur et des élèves. Rennes: Presse universitaire de Rennes. pp 13-49.

54. Sensevy, G. (2006). L'action didactique : Eléments de théorisation. Revue suisse des sciences de l'éducation. 28(2). Academic Press Fribourg. pp 205-225.

55. Terret, T. (2002). Le sport contre la santé : Les redéfinitions de l'éducation physique (1945-1960). Education et santé sous la IV ème République. Saint Etienne : Presse Universitaire. pp 11-54.

56. UNESCO. (1978). La charte internationale de l'éducation physique et du sport.

57. Van Der Maren, J. M (1996). Méthode de recherche pour l'éducation. Bruxelles : De Boëck Pédagogie et développement.

58. Vermersch, P. (1994). L'entretien d'explication en formation initiale et en formation continue. Paris : ESF.

59. Verret, M. (1975). Le temps des études. Thèse d'Etat. Université de Paris V. Paris.

60. Zinsou, G. (2011). Problématique de l'enseignement du volleyball selon l'approche par les compétences dans les lycées et collèges de Cotonou et de Porto-Novo. Mémoire de Maîtrise STAPS, PortoNovo : INJEPS-UAC. 61p.

61. Zomanhoun, A. C. (2008). Le système éducatif béninois de la période coloniale à nos jours: Une approche comparée des contenus enseignés en EPS. Mémoire de Maîtrise en STAPS. Porto-Novo : INJEPS-UAC.58p 